\section{Seeing is believing in lineages}

\section{from a Correspondent}

AT last a directly observed and accurate cell lineage is available for the development of one organism, the nematode Caenorhabditis. As reported at a recent meeting*, the lineage runs from the fertilised zygote to the completion of embryogenesis (550 cells), and then there is a gap of one or two cell divisions, which have not yet been studied, before the lineage runs directly through to adult structures. This description is now up for analysis and G.von Ehrenstein (Max-Planck Institute for Experimental Medicine, Göttingen) has isolated at least seven temperaturesensitive mutants whose maternal gene expression is necessary and sufficient for normal embryogenesis, and which eventually block development at the 'lima bean' stage. Three of these produce abnormal guts without obviously disturbing the rest of the lineage; in the gut lineage one changes the direction of a single division while the other two change the relative timing of division and migration of gut primordial cells to the interior. It is, however, unlikely that the regularity of the lineage merely depends on control of cell division rate and cell division direction for there are even more extensive cell migrations across four or five cell diameters in other parts of the embryonic lineage. Certainly there is regulation in the post-embryonic lineage for, following laser ablation of single cells, their lineage and adult structures may be replaced by neighbouring cells within an 'equivalence group' (J. Kimble, MRC Laboratory of Molecular Biology, Cambridge). It remained unclear whether the replacing cell had to repeat the lineage of the ablated cell in order to replace its adult structures because within an equivalence group it always did so. (This and related work with Caenorhabditis was mentioned in News and Views, 279, 758; 1979.)

The masterplans in 'mosaic' eggs were certainly under attack even though there are nice sets of computer simulations which could manufacture such plans (E. Parisi, Laboratory of Molecular Embryology, Naples). P. Guerrier (Biological Station, Roscoff) reviewed his own and van den Biggelaar's beautiful manipulation experiments on the 'mosaic eggs' of annelids and molluscs and made it difficult to see how anybody could still believe in mosaicism in these embryos (for example, Bigelaar \& Guerrier, Devl Biol. 68, 462; 1978). The Drosophila egg must at least be allowed its polarities, and to go with 'bicaudal', there is now a maternal effect mutant, dorsal $(d l)$, which leaves the anterior-posterior polarity intact but which lacks lateral and ventral structures

*The meeting on Cell Lineage, Stem Cells and Cell Determination was held in Seillac on 20-24 May 1979. The proceedings will be published by Elsevier-North Holland.
(C. Nüsslein-Volhard, EMBL, Heidelberg). It may be 'all dorsal' for no dorsal midline can be detected, and in order to show formally that the laser ablation fate map was altered by this dorsal hypodermis embryo it was necessary to put the mutation on a background which reduced its penetrance so that some ventral structures were formed. The polar tendencies of the Drosophila egg must arise during oogenesis and E. Weischaus (EMBL, Heidelberg) has now made use of another maternal effect mutant, $f s(I) K 10$, in a neat mosaic analysis of stem cell behaviour during oogenesis. This mutation is expressed as a chorion defect (allowing immediate recognition of affected eggs) but its expression is restricted to the germ line; consequently recombination induced by $\mathrm{X}$-irradiation during the formation of eggs can be used to follow the behaviour of stem cells which are egg precursors. From blastoderm to pupa, the stem cells increase to about 100 and this level is maintained through egg production. This technique could be exceptionally useful for analysing the effect of characters expressed in the nurse-cell oocyte clusters on subsequent development.

Even those working on haemopoietic stem cells could now see lineage diversification with their eyes and could provide direct evidence for multipotential stem cells. G.R. Johnson (Walter and Eliza Hall Institute, Melbourne) has now picked single cells, waited for them to divide twice in isolated liquid culture, separated the four cells, found that each could form colonies which contained a variety of cell types, and thus showed that one cell could form erythrocytes, macrophages and granulocytes. The implication is that cell lineages can separate without the special cell contacts and microenvironments which are supposed to pick out and diversify stem cells; nobody could decide if the behaviour of these clones was natural or perverted. The environmental lobby made a comeback with a study of the progressive education of $T$ lymphocytes to attack virus-infected cells. It has now been shown that the major histocompatibility complex (MHC) type of both the thymus and the 'post-thymic environment' educates cytotoxic $\mathrm{T}$ cells to kill virus-infected cells with similar MHC (R.M. Zinkernagel, Scripps Clinic, La Jolla).

If lymphocytes can be educated, then nerve states can be altered and stabilised. The evidence that individual post-mitotic neurones can functionally convert from adrenergic to cholinergic cells in the presence of non-neuronal cells is now convincing and P.H. Patterson (Harvard University) went on to show that this change could be prevented by depolarising the neurone. This change could be seen directly in cell culture; could it be as simple as that in the adrenergic-cholinergic switch shown by neural crest cells transplanted to different levels of the axis of developing chick-quail chimaeras? Certainly these transplants illustrate beautifully the importance of the migratory path and final location in picking out the phenotype of neural crest cells (N. Le Douarin, Embryology Institute, Nogentsur-Marne). Depolarising a neurone in culture may mimic normal neurone activity, and chronic stimulation of the spinal cord during embryogenesis may do the same. If it does, then the stabilisation of nerve synapses on muscle during development may be shown to depend on nerve activity. The system is the posterior latissimus dorsi muscle of the chick which early in development has several synapses per fibre and of these only one remains in the adult; stimulation of the spinal cord during embryogenesis maintains multiple connections (P. Changeux, Pasteur Institute, Paris). Is this the selective stabilisation theory of nerve connections in action?

\section{Mutants of the lac repressor}

\section{from Alan D. B. Malcolm}

As part of their study of the binding of the lac repressor protein of Escherichia coli to the lac operator DNA, Miller and colleagues set out several years ago to produce mutants of the repressor protein by an ingenious technique, and the culmination of their work appears in three recent papers (J. molec. Biol. 130, 191, 223 $\& 249 ; 1979)$. Miller's approach has been to use chemical mutagens to produce nonsense mutations (where a coding triplet is replaced by one of the three 'stop' signals, UAG, UAA or UGA) in a suppressor strain which possesses a tRNA able to substitute an amino acid in place of the stop. So far it is possible to insert Ser, Gln, Tyr, Lys or Leu at UAG; Gln, Tyr or Lys at UAA and Trp at UGA. This method has the advantage that it is easy to screen for proteins with minimal functional defects (this is difficult to achieve by conventional mutagenic techniques) and also that some of the mutations introduced would be expected to require two base changes. It also has some limitations. Mutations can be introduced only at positions where the wild type can be mutated into one of the three 'stop' signals. It is known that not all sites are equally susceptible to mutagenesis and the distribution of these 'hot spots' has been the subject of a previous paper (Farabaugh et al. J. molec. Biol. 126, 847; 1978). The amino acids that may be introduced are limited by the availability of suppressor strains, although there seems to be no reason why the number of these could not be increased in the future. Miller et al. have collected more than 300 mutants at 90 out of the 360 positions in the lac repressor and these can be altered in various ways: inducer binding, ability to form tetramers and binding to DNA (either operator or 